\title{
Anabases
}

ANABASES Traditions et réceptions de l'Antiquité

5 | 2007

Varia

\section{Chateaubriand et la « fièvre des ruines »}

Jean-Paul Clément

\section{OpenEdition}

Journals

Édition électronique

URL : http://journals.openedition.org/anabases/3185

DOI : 10.4000/anabases.3185

ISSN : 2256-9421

Éditeur

E.R.A.S.M.E.

Édition imprimée

Date de publication : 1 mars 2007

Pagination : 179-189

ISSN : 1774-4296

\section{Référence électronique}

Jean-Paul Clément, " Chateaubriand et la « fièvre des ruines » », Anabases [En ligne], 5 | 2007, mis en ligne le 01 janvier 2012, consulté le 20 octobre 2019. URL : http://journals.openedition.org/anabases/ 3185 ; DOI : 10.4000/anabases.3185 
Anabases 5 (2007), p. 179-189

\section{Chateaubriand et la « fièvre des ruines "}

JeAn-Paul Clément

LE 27 JUIN 1803, Chateaubriand arrive à Rome après être passé par Lyon, où il est accueilli en nouveau Père de l'Église. Rome, il ne la connaissait que d'une manière livresque, qu'il nous donne dans le Génie du Christianisme. L'extraordinaire succès de l'ouvrage, venant à point nommé illustrer de manière flamboyante la politique concordataire du Premier Consul, l'épître dédicatoire adressée au restaurateur des autels - « La France agrandie par vos victoires a placé en vous son espérance, depuis que vous appuyez sur la Religion les bases de l'État et de vos prospérités »-, portent jusque sur les rives du Tibre l'homme des septentrions, qui avait attendu un an avant d'être nommé auprès du cardinal Fesch, nouvel ambassadeur après douze ans de rupture avec le Saint-Siège.

La lumière de Rome, la beauté des monuments, les méditations que lui inspirent les ruines, font de cette ville sa "seconde patrie ». Sous l'empire de l'exaltation, négligeant d'attendre le cardinal Fesch, son chef de poste, il demande audience au pape, l'obtient, se présente et reçoit un accueil qui met le comble à ses transports (2 juillet). Pie VII, « le pontife des tribulations, figure pâle et triste mais grande et digne ", vient au-devant de lui, lui prend la main, le fait asseoir à ses côtés, l'appelle son "cher Chateaubriand ", lui parle en termes flatteurs de son livre, ouvert sur sa table avec un signet. Mais les initiatives intempestives de Chateaubriand, ses relations avec les royalistes exilés, sa visite au roi abdicataire de Sardaigne que les troupes françaises venaient de renverser, suscitent la suspicion. Le sort malheureux de sa maitresse, Pauline de Beaumont, atteinte de phtisie et traversant la France en agonie pour venir mourir entre ses bras après une ultime visite au Colisée - « [...] c'était un de ces jours d'octobre, tels qu'on n'en voit qu'à Rome. [...] Elle leva les yeux ; elle les promena lentement sur ces portiques morts eux-mêmes depuis tant d'années, et qui avaient vu tant mourir ; les ruines étaient décorées de ronces et d'ancolies safranées par l'automne, et noyées dans 
la lumière. La femme expirante abaissa ensuite, de gradins en gradins jusqu'à l'arène, ses regards qui quittaient le soleil ; elle les arrêta sur la croix de l'autel, et me dit : "Allons ; j'ai froid. " Je la reconduisis chez elle ; elle se coucha et ne se releva plus ${ }^{1}$ " - avait, il est vrai, attendri le Sacré collège, et même l'ambassadeur, qui envoya ses carrosses pour suivre le cortège funèbre. Mais l'hostilité restait vive. Chateaubriand, dépourvu de tout pouvoir, relégué dans les combles du palais Lancelotti, où les puces lui montaient jusqu'aux genoux, ne pouvait persister dans une place où il se sentait rejeté. Il demanda son rappel, partit pour Naples, Pompéi, Herculanum, recueillant toutes les images qui nourriront son roman épique Les Martyrs.

Rentré à Paris, il est nommé - un peu par dérision - auprès de la République toute virtuelle (elle n'aura que deux ans d'existence avant de devenir le département du Simplon) du Valais. L'exécution du duc d'Enghien, de sang royal, petit-fils du prince de Condé, qui avait commandé l'armée des Princes au début de la Révolution, provoque chez lui un retour - qu'avait préparé le Génie du Christianisme - à l'histoire, à la grandeur d'une dynastie et à sa déchéance. Il rompt. Les relations avec Bonaparte devenu Napoléon et Chateaubriand sont extrêmement complexes, Napoléon souhaitant à certain moment attirer Chateaubriand autour de lui, non pas en tant qu'homme politique, mais en tant que génie littéraire, en le faisant entrer de force à l'Institut, mais faisant exécuter sans scrupules le cousin de Chateaubriand. Désormais, Chateaubriand entre dans une opposition active, et ardente lorsqu'il publiera en avril 1814 De Buonaparte et des Bourbons, ou Des raisons de se rallier à nos princes légitimes.

Si nous poursuivons notre fil chronologique avant d'entrer dans le thème proprement dit, il faut franchir vingt-cinq ans. En 1828, Chateaubriand est couvert de gloire lorsqu'il arrive à Rome par petites étapes, partout salué par les autorités : pair de France, ancien ministre des Affaires étrangères (avec à son crédit la réussite de la guerre d'Espagne de 1823), journaliste et pamphlétaire redouté, " tombeur " du gouvernement ultra de Villèle - ce que Charles X ne lui pardonne pas. Chevalier des Ordres du Saint-Esprit, il a reçu de tous les souverains d'Europe des décorations prestigieuses : la Toison d'or, l'Ordre de Saint-André de Russie, l'Aigle noir de la Prusse, la Très Sainte Annonciade, l'Ordre du Christ du Portugal, etc. Il espère un moment être nommé aux Affaires étrangères une fois encore. Le roi s'y oppose. Il est aussi dangereux au gouvernement que dans l'opposition. On lui offre une ambassade, un " exil doré " (9 octobre 1828 - 16 mai 1829). Il accepte, non sans quelque réticence. Mais l'attrait de Rome est toujours aussi fort. De même qu'il se complut à se rappeler le jeune émigré miséreux qui avait abordé les côtes anglaises en 1793 lorsque, en 1822, ambassadeur de France de Sa Majesté Très Chrétienne à Londres, il se rendait à quelque brillant raout donné par l'aristocratie anglaise, en 1828 Chateaubriand se souvient de ses médiocres débuts diplomatiques à Rome.

1 Chateaubriand, Mémoires d'outre-tombe, éd. Jean-Paul Clément, Paris, Gallimard, coll. «Quarto », 1997, livre XV, chapitre 4, t. I, p. 871-872. 
Il quitte Paris pour rejoindre une ambassade brillante, où il entend faire ce que Fesch n'avait pas fait : être le digne successeur du cardinal de Bernis, dernier ambassadeur de Louis XVI, surnommé "le roi de Rome "; faire de l'ambassade, au palais Simonetti, « l'auberge de l'Europe ». Il avance à petites étapes, guetté par les autorités au seuil des villes, logé dans des palais, vers l'arène du monde qu'il avait découverte sous le triple signe constamment mêlé de l'émerveillement, de la mort et de l'amour pour Juliette Récamier. Chateaubriand donne des fastes à l'ambassade mais délaisse son palais. Il invite ses convives à la Villa Médicis (sur le Pincio), siège de l'Académie de France à Rome, dirigée alors par Horace Vernet, bonapartiste libéral, attaqué par les zelanti pro-autrichiens à Rome et les ultras à Paris, et que Chateaubriand soutient tout en ayant fait venir son père pour surveiller le fils, qui vit comme " au bivouac ", " un serpent autour du cou ". Sur le Pincio, où fleurissent les roses, où les chênes verts tamisent les rayons de lumière, il goûte un plaisir subtil. Il sent vivre dans son esprit le contraste entre les persistances de la nature et la brièveté des êtres, cette antithèse qui est au fond même de la vie et que les poètes romantiques ont comme découverte. Sous cette impression aiguë, il dit : «[...] je ne m’étais pas douté de ce que pouvaient être des fêtes à Rome : elles ont quelque chose de la poésie antique qui place la mort à côté des plaisirs ${ }^{2}$."

Chateaubriand s'intéresse aux artistes. Il écrit : «Je voudrais être né artiste : la solitude, l'indépendance, le soleil parmi des ruines et des chefs-d'œuvre, me conviendraient. [...] Ma vie a été misérablement accrochée aux buissons de ma route ; heureux si j'avais été l'oiseau libre qui chante et fait son nid dans ces buissons ${ }^{3}$ ! "Car toutes les existences différentes de la sienne, et qui ne l'auraient pas davantage assouvi, le tentent. Il essaie d'approcher de ces destinées étrangères : " Pinelli, entre deux ivresses, écrit-il, m'a promis douze scènes de danses, de jeux et de voleurs. C'est dommage qu'il laisse mourir de faim son grand chien couché à sa porte ${ }^{4}$. » Pas plus que Chateaubriand le Diario di Roma ne laisse ignorer cette généreuse commande. Le fameux graveur ne devait pas faire douze eaux-fortes inédites pour l'ambassadeur, mais les prendre parmi ses nombreuses ouvres déjà exécutées, pleines de verve et de pittoresque. Chateaubriand élève à San Lorenzo in Lucina une stèle à la mémoire de Nicolas Poussin, celui qui a le mieux compris la campagne romaine, qui est " tout romain ", tout comme lui-même. Il confie l'ouvrage à de jeunes élèves de la Villa Médicis, Vaudoyer, Louis Desprez et Lemoine. Quand on parle d'élever un monument au Tasse, il est l'un des souscripteurs. Pourquoi ? Une ligne à Mme Récamier répond, évocatrice d'amour et discrètement confiante : "L'homme que vous aimez le plus, dites-vous, après moi, Le Tasse. " À Saint-Onuphre, Chateaubriand pense un moment finir ses jours à côté de la cellule où mourut le poète, à l'ombre des orangers. Poussin et Lorrain 
ont passé au Capitole, songe-t-il, et il ajoute en un alexandrin d'une dédaigneuse magnificence : «Des rois y sont venus et ne les valaient pas. » Une fois de plus, il aspirait à Rome à se rapprocher de ses favoris par d'indéfinissables métempsychoses. Avoir été le contemporain de tous les hommes qui l'attirent dans les siècles divers, quel rêve irréalisable !...

Les activités de Chateaubriand n'épuisaient pas toutes les ressources de sa fougue. Lartiste qui avait chanté en un jour de jeunesse les beautés sobres de la campagne romaine n'était pas mort. Sous la terre qui l'a célébré, Chateaubriand recherche les vestiges du passé. Il entreprend, avec Visconti jeune, des fouilles à Torre Vergata, dans l'endroit « le plus beau et le plus désert ». Plusieurs fois par semaine, il court au chantier. Devant le squelette présumé d'un Gaulois, il déclame sur les jeux bizarres de la destinée ; il s'écrie : "C'étaient des géants que nos aïeux ; [...] nous sommes des pygmées en comparaison. » Il passe l'anneau de l'ancêtre à son compagnon, qui tente, mais en vain, d'y glisser le doigt et remarque doucement : " Je n'ai pourtant pas sept pieds. " Mais de bonne grâce, l'écrivain reconnaît sa défaite : « Méchant garçon que vous êtes! je ne vous amènerai plus ici avec moi, vous avez gâté toute ma tirade. "

Bien qu'il ait, au début, marqué une certaine froideur envers la Rome antique qui l'avait enthousiasmé, se souvenant de la pureté des lignes attiques des temps de son voyage en Grèce de 1806-1807, Chateaubriand est vite repris par la " fièvre des pierres ». Au milieu des vicissitudes, l'admiration pour Rome et la campagne qui l'environne demeure. Elle relie deux époques. «Venez donc ici si vous voulez être étonné, si vous voulez savoir en trois jours ce que vous n'apprendriez pas en trois siècles dans les livres, si vous voulez [...] vous rassasier des plus grands souvenirs. [...] Je puis vous assurer, mon cher Mathieu, qu'on n'a rien vu quand on n'a pas vu Rome ${ }^{5}[\ldots]$ ", écritil à Mathieu Molé le 16 juillet 1803. À l'époque, Chateaubriand courait le jour du Colisée à la colonne Trajane, du château Saint-Ange au Panthéon, assistait à l'office à Saint-Pierre. Son enthousiasme est confirmé alors par Artaud de Montor, son prédécesseur :

Je me chargeai d'être le cicerone à Rome du nouveau secrétaire : je le conduisis par la rue détournée qui est à droite de la rue du Borgo, jusqu'à la façade de Saint-Pierre, pour qu'il eût tout à coup la surprise de ce coup d'œeil, et j'eus le plaisir de jouir de ses émotions qu'il exprimait d'une manière simple, franche et en même temps imprévue. Il parlait peu, parce qu'il était comme hors de lui d'admiration. Sans doute rien de si grand, de si magnifique n’avait frappé ses yeux ; il paraissait ravi de contempler ainsi le plus beau temple de notre Culte 6 .

5 Chateaubriand, Correspondance générale, éd. Pierre Riberette, Paris, Gallimard, 1977, t. I, p. 237.

6 Artaud de Mentor, Histoire du pape Pie VII, Paris, Le Clere, 1836. 
En 1828, Chateaubriand a vieilli, lui qui déteste la vieillesse ; son cœur est vert, mais ses membres sont gagnés par des rhumatismes : "Quand je me promène seul à présent au milieu de tous ces décombres des siècles, ils ne me servent plus que d'échelle pour mesurer le temps : je remonte dans le passé, je vois ce que j'ai perdu 7 [...]. » Puis la « déplaisance " se dissipe. Il finit par " adorer ce qui [l'] avait laissé froid d'abord ». Il reprend ses déambulations à travers la campagne romaine, "écoute le silence », "regarde passer son ombre de portiques en portiques "; il la connait si intimement, jusqu'au plus petit chemin entre deux haies. Le rapprochement avec Combourg vient naturellement sous sa plume, et devient un fragment d'une belle et grande patrie où il se sent à l'aise : celle des souvenirs. Il court de nouveau le long du Tibre, jette un regard sur le château Saint-Ange, assiste à l'office de Saint-Pierre, reconnaît le Vatican, flâne au clair de lune, contemple du haut de la Trinité des Monts « les édifices lointains qui paraissent comme les ébauches d'un peintre ou comme les côtes effumées vues de la mer du bord d'un vaisseau ». Tous les plans s'adoucissent; les objets s'arrondissent, la lumière s'insinue dans la masse obscure des rochers et des feuilles. Rome continue d'exercer une puissante attraction. Sainte-Beuve nous rapporte le récit de la rencontre d'Ampère et de Chateaubriand chez Mme Récamier, peu de temps après son retour en 1829 :

À peine la main serrée, ce furent des nouvelles sans fin de la Ville éternelle : et les fouilles et le Colisée, et ce bouquet d'arbres proche Saint-Jean-de-Latran, et la découverte si belle et si imprévue de la campagne au sortir de la porte Saint-Pancrace ; et ces ruines sans nom, entassées, dites Roma vecchia !... M. de Chateaubriand se rappelait tout, il racontait ses promenades dans ces plaines austères dont il sait chaque butte, chaque repli autant et mieux que pour notre plaine de Montrouge. J'écoutais, voyant dans ces grands récits l'image exacte des lieux témoins des choses immortelles ${ }^{8}$.

Las, le mouvement des sociétés est inéluctable, affirme l'homme des réalités : tout change, les fleuves ne remontent pas à leurs sources. L'homme des chimères le contredit :

Une compagnie étrangère est venue cet hiver (1829) proposer le défrichement de la campagne romaine : ah! messieurs, grâce de vos cottages et de vos jardins anglais sur le

7 Mémoires d'outre-tombe, livre XXX, chapitre 10, t. II, p. 2003. « Les plaisirs de la jeunesse reproduits par la mémoire, écrit-il ailleurs, sont des ruines vues au flambeau " (ibid., livre XII, chapitre 5, t. I, p. 730).

8 SAINTE-BEuve, Chateaubriand et son groupe littéraire sous l'Empire, éd. Maurice Allem, Paris, Classiques Garnier, 1948, t. I, p. 328. Ampère, ami de Chateaubriand et familier de l'Abbaye-aux-Bois, décrira Rome en 1835. Le tableau est le même : "Ce qui me plaît [...], ce sont les quartiers déserts, les monuments abandonnés, les vignes couvrant les fûts des colonnes renversées, les buffles dans le Forum, et surtout les fragments antiques enfouis dans l'architecture moderne : l'architrave d'un temple servant de linteau à une porte d'église ; un tronçon de colonne faisant l'office d'une borne au coin d'une rue, des échoppes nichées sous les gradins du théâtre de Marcellus, ou de petites maisons penchées sur les tombeaux de la voie appienne.» 
Janicule! si jamais ils devaient enlaidir les friches où le soc de Cincinnatus s'est brisé, sur lesquelles toutes les herbes penchent au souffle des siècles, je fuirais Rome pour n'y remettre les pieds de ma vie ${ }^{9}$.

Chateaubriand s'est voulu le peintre, l'inventeur - et il n'en était pas peu fier - de la «campagne romaine ». Le Président de Brosses n'avait vu qu'une plaine aride et déserte. Lalande s'étonne de la nudité des lieux, "plaine aride et brûlante ", de la chaleur du climat. Dupaty, dans son Voyage en Italie, à la veille de la Révolution, ne voit que " des montagnes décharnées, sur des rivages où la mer est seule ». Gilbert Burnet insiste sur les dangers que présente cette plaine marécageuse, "vapeurs puantes qui corrompent l'air des environs ». Pour Bonstetten, la race humaine semble finir avec le Capitole. Goethe, qui se promenait le soir au clair de lune, le décrit dans son Voyage en Italie. On sait que Goethe fit deux séjours à Rome : l'un du 1er novembre 1786 au 21 février 1787, l'autre du 6 juin 1787 à la mi-avril 1788. Il en rapporta son Voyage en Italie et ses Élégies romaines. Mais il n'a presque pas visité la campagne. Il n'est allé ni à Tivoli, ni à Albano. Avec lui, tous les voyageurs allemands avaient chanté Rome et ses monuments. Avec les romantiques, il avait exprimé le sentiment de la plus profonde mélancolie que lui inspiraient les ruines du Colysée, la Villa Pamphili... Chateaubriand a donné aux lettres françaises l'interprétation la plus belle et la plus profonde des ruines et de la campagne romaine, en lui donnant un sens et une profondeur tout particuliers. Dans ce décor qu'animent ses humanités, les monuments qu'il chérit surtout sont les colonnades et les aqueducs. D'arche en arche, de pilier en pilier, il en poursuit le ressaut qu'il regarde approfondir l'espace pour mieux éprouver ce sentiment du vide et du désert qu'aucun autre avant lui n’avait éprouvé. La campagne romaine représente le temps mais aussi le vide, la béance de l'histoire. Dans le Congrès de Vérone, se rendant à son ambassade de 1828, Chateaubriand écrit : «Entre mes voyages, séparés seulement de quelques années, il y avait de moins une monarchie de quatorze siècles. " Rome tout à la fois évoque un présent qui le ranime, mais le passé fait surgir en lui un passé encore antérieur, aboli, qu'il ravive. À Rome, Chateaubriand éprouve une obsession : les ruines se faisant écho d'époque en époque, les conclaves où « des vieillards se transmettent une royauté élective qui expire bientôt avec eux ", sépulcres exhumés " qui offrent le spectacle d'une résurrection et pourtant n'attendent qu'une mort plus profonde ». Tout dans la grande nécropole universelle signale cendre et poussière, poussière de poussière, néant indéfiniment démultiplié.

Rome et sa campagne, c'est aussi ce débat contre le temps. "Que ne suis-je, écrit Chateaubriand dans les Mémoires, entre les mains [...] de ce grand dévorateur de siècles que je croyais arrêtés, de ce temps qui me fait pirouetter dans les espaces avec lui. »Que le tourbillon s'apaise, que les souvenirs s'ordonnent, tel est l'objet des Mémoires d'outretombe, de la correspondance à Mme Récamier. La campagne romaine, c'est à la fois la 
réunion du désert, du silence, du retrait, et ces ruines qui se dressent en témoins d'un grand passé. Ce ne sont plus les vieux donjons ruinés de Bretagne, mais la ruine que les peintres avant les écrivains ont su au XVIII siècle, par l'œuvre de Piranèse, admirablement décrire. Certes on trouve dans ces ruines les signes picturaux chers à Chateaubriand : les arbres, les clochers, les colonnes, mais la forme de ces ruines est rompue. Élancées, elles sont la fantaisie même, pittoresque, tout à la fois réunissant en leur sein la vie et la mort, brisées mais vivantes, surprenantes de par leur structure, incongrues mais naturelles et comme issues du sol dont elles seraient l'unique végétation. Belles comme une esquisse, les ruines sont le repère parfait. "Les ruines ont [...] des harmonies particulières avec leurs déserts, selon le style de leur architecture, les lieux où elles sont placées, et les règnes de la nature au méridien qu'elles occupent. Dans les pays chauds, peu favorables aux herbes et aux mousses, elles sont privées de ces graminées qui décorent nos châteaux gothiques et nos vieilles tours ; mais aussi de plus grands végétaux se marient aux plus grandes formes de leur architecture ${ }^{10}$. "Ces ruines, Chateaubriand les avait déjà évoquées dans le Génie du Christianisme, avant que l'expérience de 1803 ne vienne nourrir quelques-uns des grands chapitres des Martyrs. Il distinguait alors deux sortes de ruines : celles des hommes, images du néant - ainsi les ruines modernes s'identifient-elles à la Révolution -, et les ruines ouvrages de la nature :

[...] l'une, ouvrage du temps ; l'autre, ouvrage des hommes. Les premières n'ont rien de désagréable, parce que la nature travaille auprès des ans. Font-ils des décombres, elle y sème des fleurs; entrouvrent-ils un tombeau, elle y place le nid d'une colombe : sans cesse occupée à reproduire, elle environne la mort des plus douces illusions de la vie ${ }^{11}$.

Faite de plein et de creux, la ruine se dessine sur l'horizon d'un bleu effumé. Chateaubriand l'imagine tournée vers le dehors, inerte certes de par sa substance mais encore toute vibrante, murmurante, bourdonnante tout autant que forêts, oiseaux, feuillages. S’il n'a pas inventé la campagne romaine, il lui a donné tout son sens. La ruine est tout à la fois rêverie du siècle et caprice végétal qu'il évoque : chaud effritement des pierres rouges, vibratilité de quelque mousse, graminée ou herbe folle, mariage de la matière morte, à la fois matière et passé, et se ranimant, revivant avec l'humble végétal pour s'éparpiller à travers lui dans la légèreté de l'atmosphère. Ruines chrétiennes dans le Génie du Christianisme, ruines romaines dans les Mémoires, mais partout le vent circule. La ruine lie son image à la mélancolie du révolu, au thème de l'oubli, de l'effacement universel, et donc à l'intuition d'un présent fantôme, d'une vie éprouvée comme fragilité et comme songe. Jean Starobinski, dans L'invention de la liberté, écrit :

10 Génie du Christianisme, III partie, livre V, chapitre V, éd. Maurice Regard, Paris, Gallimard, Pléiade, 1978, p. 884.

Ibid., p. 882. 
La poésie de la ruine est poésie de ce qui a partiellement survécu à la destruction, tout en demeurant immergé dans l'absence : il faut que personne n'ait gardé l'image d'un monument intact. La ruine par excellence signale un culte déserté, un dieu négligé. Elle exprime l'abandon et le délaissement. Le monument ancien était un mémorial, [...] il perpétuait un souvenir. Mais le souvenir a été perdu, une signification seconde lui succède, annonçant dorénavant la disparition du souvenir que le constructeur avait prétendu perpétuer dans la pierre. Sa mélancolie réside dans le fait qu'elle est devenue un monument de la signification perdue.

Ce sont les bienfaits de cette mélancolie que Chateaubriand éprouve devant les ruines de Rome après les avoir éprouvés en Grèce. Et c'est pour cela qu'il les chérit. Leur réalité se fonde sur l'évidence d'un manque de réalité. Naturelle, formée par la lente érosion du temps, la ruine semble avoir le pouvoir de nous entrainer hors d'elle-même, vers une sorte d'au-delà indéfini. Phrase admirable : "Un débris de tour témoigne d'un autre temps, comme la nature accuse ici des siècles immémorés. " La ruine est également symbolique. Le " moi " de Chateaubriand est cet homme mourant même à chaque minute de sa vie. Les hommes pour Chateaubriand sont bien des ruines, et lui tout le premier. Il écrit : " Je sais mes ruines. » Et le 11 avril 1829, quelques jours avant Pâques, il peut écrire de Rome à Juliette Récamier : "Toutes les ruines semblent rajeunir avec l'année », pour conclure presque allègrement : "Je suis du nombre. »

Pour Chateaubriand, un site ne vaut que par les souvenirs ${ }^{12}$. Or, depuis le tombeau de Scipion jusqu'à celui de Virgile, toutes les gloires sont ensevelies à Rome ; la solitude succède au tumulte d'autrefois ; le Tibre court presque inconnu dans ses rives abandonnées. Une idée le poursuit pas à pas, le néant des choses humaines. À Fontanes, destinataire de la Lettre sur la campagne romaine du 10 janvier 1804, il écrit dès les premières lignes : « [...] je vais [...] essayer de vous donner une idée générale des dehors de Rome 13 [...]. » La relisant vingt-cinq ans plus tard, il remarque que d'autres auteurs l'ont suivi dans cette voie d'admiration et observe : «[... j'avoue que je l'ai trouvée d'une telle exactitude qu'il me serait impossible d'y retrancher ou d'y ajouter un mot ${ }^{14}$. " La Lettre, plus encore que Corinne, de Germaine de Staël, connaîtra un retentissement immense, influencera Lamartine, Stendhal même (qui parle de la campagne

$12 C f$. lettre à Mme de Staël, $1^{\text {er }}$ septembre 1805, Correspondance générale, t. I, p. 368-369, qui paraîtra dans le Mercure de France, le 3 mars de la même année. Correspondance générale, t. I, p. 298.

14 Mémoires d'outre-tombe, livre XXX, chapitre 9, t. II, p. 2002. Rome avait en effet peu changé, grâce à l'incurie de l'administration pontificale. Chateaubriand s'en réjouit. Il fait imaginer ce que cette ville - " le délaissé de la cité " - recelait de beauté, de force poétique et de mélancolie. Chateaubriand n'a pas inventé, tout juste embelli, sur les traces de Poussin et de Claude Lorrain. "Mon autre compatriote Claude mourut aussi sur les genoux de la reine du monde. Si Poussin reproduit la campagne de Rome lors même que la scène de ses paysages est placée ailleurs, le Lorrain reproduit les ciels de Rome lors même qu'il peint des vaisseaux et un soleil couchant sur la mer » (ibid., livre XXX, chapitre 6, t. II, p. 1984). 
romaine en des termes proches de la Lettre, mais qu'il ignore superbement). Chateaubriand se flatte d'avoir devancé Byron : « Les Martyrs et ma Lettre [...] ont l'inappréciable avantage pour moi, d'avoir deviné les inspirations d'un beau génie ", dit-il joliment, comme on donnerait un coup de patte, toute griffe cachée. On pourrait citer aussi Shelley, W. von Humboldt, et, chez les peintres, Clérisseau, Granet, Joseph Vernet et Corot. Certes on ne peut passer sous silence l'école picturale " ruiniste » : Hubert Robert, Pannini et leurs paysages fictifs, le graveur Piranèse. Mais la comparaison s'arrête là. Chez Piranèse, qui ouvre la voie au roman noir anglais, la démarche est différente de celle de Chateaubriand. À la fin du Moyen Âge, l'Europe du Nord subissait une vague morbide de la représentation du cadavre humain dévoré par les vers; expression d'une tradition religieuse toute tournée vers la méditation sur les misères de l'homme et vers le mépris des choses humaines. Avec Piranèse, les splendeurs de plusieurs siècles de civilisation italienne se concluaient en une vision tragique. Ce qui avait été à la fin du Moyen Âge un sentiment tragique de la caducité de l'homme, fut chez Piranèse un sens tragique de la caducité des édifices, de la mort des monuments. C'est une élégie dramatique de la ville ancienne, mais aussi les premiers balbutiements de la ville moderne. En effet, des ruines colossales naitront les colonnes géométriques des villes modernes. Piranèse est le premier artiste à donner l'idée d'une métropole faite plus à la mesure des titans qu'à la mesure de l'homme.

Dans sa Lettre à Fontanes, qui suit de deux ans la vaste fresque du Génie, Chateaubriand se livre à une célébration du " paysage d'histoire et d'art ". Comme l'ancienne France chrétienne menacée de disparition, Rome aux yeux du peintre-poète est un tout, un autre chef-d'œuvre en péril, dont il importe de fixer la forme intacte par la description, pour la faire aimer et pour la sauver. L'architectonique naturelle de son site est en correspondance harmonique avec la splendeur architecturale de la cité :

Rien n'est comparable pour la beauté aux lignes de l'horizon romain, écrit-il, à la douce inclinaison des plans, aux contours suaves et fuyants des montagnes qui le terminent. Souvent les vallées dans la campagne prennent la forme d'une arène, d'un cirque, d'un hippodrome ; les coteaux sont taillés en terrasses, comme si la main puissante des Romains avait remué toute cette terre. Une vapeur particulière, répandue dans les lointains, arrondit les objets et dissimule ce qu'ils pourraient avoir de dur ou de heurté dans leurs formes. Les ombres ne sont jamais lourdes et noires; il n'y a pas de masses si obscures de rochers et de feuillages, dans lesquelles il ne s'insinue toujours un peu de lumière. Une teinte singulièrement harmonieuse, marie la terre, le ciel, et les eaux : toutes les surfaces, au moyen d'une gradation insensible de couleurs, s'unissent par leurs extrémités, sans qu’on puisse déterminer le point où une nuance finit et où l'autre commence. Vous avez sans doute admiré dans les paysages de Claude Lorrain, cette lumière qui semble idéale et plus belle que nature ? eh bien, c'est la lumière de Rome ${ }^{15}$ !

15 Lettre à $M$. de Fontanes sur la campagne romaine, éd. Jean-Maurice Gautier, Genève, Droz, 1951, p. 6-7. 
Cette lecture à la fois architecturale et luministe du cadre naturel de Rome suppose une familiarité acquise au Louvre, avec les paysages de Poussin et de Lorrain, modèles pour les peintres paysagistes contemporains : un Valenciennes, un Michallon. Chateaubriand était d'autant mieux préparé à comprendre les classiques français du " paysage historique " qu'il s'était lui-même livré à la pratique du dessin de paysage, à Londres et à Beccles, peut-être même, selon la thèse aujourd'hui contestée d'Alice Poirier, parmi des élèves de Constable et de Turner. Il avait tiré les premières leçons de cette expérience anglaise dans une Lettre sur les paysages qui date de 1795. Les " paysages " du Génie, dont la Lettre à Fontanes est un supplément (le Génie, et pour cause, évoquait fort peu Rome), font se croiser pour la première fois l'école anglaise et l'école française du paysage, le sentiment de la lumière de l'une, le sentiment de l'espace de l'autre.

Rome, ses faubourgs, ses jardins, ses ruines, ses cloîtres, sont propices à l'essor de son imagination, font vibrer sa lyre intérieure. Chateaubriand ne hante les musées que les jours de pluie. Cette "seconde patrie » tient de la réalité et du songe ; elle aiguise en lui cette dilection esthétique et morale dont les peintures de Nicolas Poussin et de Claude Lorrain donnent des représentations "idéales et plus belles que nature ». Il la voue à ne pas changer. Elle doit demeurer pour lui un témoignage d'humanité, de grandeur et de dignité, un témoignage de haute école.

Plus que toute autre ville, elle comporte - je cite - « sa partie morale et intellectuelle ». Elle fait toucher à Chateaubriand à la vanité du monde lorsqu'il contemple tous ses " colosses de gloire » évanouis dans la poussière : "Qu'arriva-t-il, il y a dix-huit siècles, à pareille heure et aux mêmes lieux ? écrit-il. Quels hommes ont ici traversé l'ombre de ces obélisques après que cette ombre eut cessé de tomber sur les sables d'Égypte ? Non seulement l'ancienne Italie n'est plus, mais l'Italie du Moyen Âge a disparu. Toutefois, la trace de ces deux Italies est encore marquée dans la Ville éternelle : si la Rome moderne montre son Saint-Pierre et ses chefs-d'œuvre, la Rome ancienne lui oppose son Panthéon et ses débris ; si l'une fait descendre du Capitole ses consuls, l'autre amène du Vatican ses pontifes. Le Tibre sépare les deux gloires : assises dans la même poussière, Rome païenne s'enfonce de plus en plus dans ses tombeaux, et Rome chrétienne redescend peu à peu dans ses catacombes ${ }^{16}$. " " J'achève souvent le tour des murs de Rome à pied ; en parcourant ce chemin de ronde, je lis l'histoire de la reine de l'univers païen et chrétien écrite dans les constructions, les architectures et les âges divers de ces murs ${ }^{17}$ " (Saint-Pierre-aux-Liens).

Peintre des ruines, à la fois corps inertes et changeants, sensible au renouvellement des saisons, rajeunissant avec elles, Chateaubriand aime le chaud effritement des pierres rouges. Mais il y superpose toujours la légèreté végétale, alliance pour lui essentielle. Au tombeau de Cecilia Metella, où il herborise, Chateaubriand s'avise que « le réséda ondé 
et l'anémone apennine font un doux effet sur la blancheur de la ruine et du sol ». Le paysage italien devient son propre paysage, son paysage intérieur, sur lequel viennent se broder des motifs de souffrance, les réminiscences de ses vies antérieures. En 1811, il s'exclamera : "Si j'étais riche - que de fois il fera entendre ce refrain - [...] l'Italie me verrait tous les deux ans, et peut-être finirais-je par me fixer au milieu des ruines de Rome 18 ", là où " les cyprès ressemblent à des femmes de l'Orient ". Mais, chrétien, c'est par la Rome des papes que Chateaubriand achève son voyage. Assistant à l'Office des Ténèbres, dans la Chapelle Sixtine, il décrit dans une lettre à Juliette Récamier cette clarté qui meurt par degré, ces ombres qui enveloppent peu à peu les merveilles de Michel-Ange et la fumée blanche qui monte de la lumière étouffée des cierges. "C'est, écrit-il, une belle chose que Rome pour tout oublier, pour mépriser tout et pour mourir. "

Les souvenirs romains devaient nous réserver une dernière méditation. Revenant de Prague, un soir de juin 1833, Chateaubriand s'assoupit dans sa calèche. C'est la fameuse prosopopée où il s'adresse à Cynthie, "fantôme d'amour " : "Qu'elle est admirable, cette nuit, dans la campagne romaine ! La lune se lève derrière la Sabine pour regarder la mer ; elle fait sortir des ténèbres diaphanes les sommets cendrés de bleu d'Albano, les lignes plus lointaines et moins gravées du Soracte. [...] Législatrice du monde, Rome, assise sur la pierre de son sépulcre, avec sa robe de siècles, projette le dessin irrégulier de sa grande figure dans la solitude lactée ${ }^{19}$. " Et j’achèverai par ce mot : "[...] je me sens de l'attrait dans les siècles divers !" s'exclame Chateaubriand, qui ajoute avec humour : "Mais il m'eût fallu ressusciter trop souvent ${ }^{20}$."

\begin{tabular}{l} 
Jean-Paul CLÉMENT \\
\hline Ancien professeur à l'IEP de Paris \\
87, rue Chateaubriand \\
F-92290 Châtenay-Malabry \\
pclement@cg92.fr
\end{tabular}

18 Lettre à Fraser Frisell, 10 mai 1811, Correspondance générale, t. II, p. 106.

19 Mémoires d'outre-tombe, livre XXXIX, chapitre 5, t. II, p. 2687.

20 Ibid., livre XXX, chapitre 6, t. II, p. 1984. 